\title{
The convex hull of the integer points in a large ball
}

\author{
Imre Bárány ${ }^{1, \star}$ David G. Larman ${ }^{2}$ \\ 1 Mathematical Institute of the Hungarian Academy of Sciences, POB 127, 1364 Budapest, Hungary \\ (e-mail: barany@math-inst.hu) \\ ${ }^{2}$ Department of Mathematics, University College London, Gower Street, London WC1E 6BT, UK \\ (e-mail: dgl@math.ucl.ac.uk)
}

Received: 28 March 1997 / Revised version: 23 March 1998

Mathematics Subject Classification (1991): 52B20, 52A27, 11P21

\section{The main result}

The "integer convex hull" of $r B^{d}$, the ball of radius $r$ centred at the origin, is, by definition

$$
P_{r}=\operatorname{conv}\left(Z^{d} \cap r B^{d}\right),
$$

which is clearly a convex polytope. How many vertices does $P_{r}$ have? Motivation for the question comes from different sources: integer programming (cf. [CHKM] $[\mathrm{BHL}])$, classical enumeration problems ([J],[Sch], or more generally [W],[Vin]), and from the theory of random polytopes (see later). For the case $d=2$ it is shown in $[\mathrm{BB}]$ that

$$
0.33 r^{2 / 3} \leq f_{0}\left(P_{r}\right) \leq 5.55 r^{2 / 3}
$$

where $f_{k}(P)$ denotes the number of $k$-dimensional faces of the polytope $P$. The limit, as $R \rightarrow \infty$, of the average of $r^{-2 / 3} f_{0}\left(P_{r}\right)$, on an interval $[R, R+H]$, is determined by Balog and Deshoullier [BD], and turns out to be $3.453 \ldots,(H$ must be large). Our main result extends (1.1) to any $d \geq 2$ and to any $f_{k}\left(P_{r}\right)$ with $k=0, \ldots, d-1$.

Theorem 1. For every $d \geq 2$ there are constants $c_{1}(d)$ and $c_{2}(d)$ such that for all $k \in\{0, \ldots, d-1\}$

$$
c_{1}(d) r^{d \frac{d-1}{d+1}} \leq f_{k}\left(P_{r}\right) \leq c_{2}(d) r^{d \frac{d-1}{d+1}} .
$$

\footnotetext{
* Partially supported by Hungarian Science Foundation Grant T 016391, and Research Grant 96$31 / 13$ of the Academy. Part of this research was carried out while this author was visiting, with an ESPRC grant, the Department of Mathematics at University College London whom he thanks for their hospitality.
} 
Using Vinogradov's $\ll$ notation this can be written as $r^{d \frac{d-1}{d+1}} \ll f_{k}\left(P_{r}\right) \ll$ $r^{d \frac{d-1}{d+1}}$. Here the implied constants depend only on dimension $d$; we will keep to this as a convention throughout the paper (unless stated otherwise).

It is the authors' conviction that lattice points and random points, in relation to convex bodies in "general position", behave similarly. Theorem 1 is another confirmation: (1.2) is in complete analogy with random polytopes. To see this, choose $n=\left\lceil r^{d} \operatorname{Vol} B^{d}\right\rceil$ random, independent, and uniform points from $B^{d}$, and let $K_{n}$ denote their convex hull. Then, according to [BL] and [B], $n^{\frac{d-1}{d+1}} \ll$ $E f_{k}\left(K_{n}\right) \ll n^{\frac{d-1}{d+1}}$, where $E$ stands for expectation. But $n^{\frac{d-1}{d+1}} \ll r^{d \frac{d-1}{d+1}} \ll n^{\frac{d-1}{d+1}}$, showing that the convex hull of $n$ random points and the convex hull of the $n$ lattice points lying in $r B^{d}$ have the same number of $k$-dimensional faces.

\section{The upper bound}

The upper bounds in (1.2) follows from a result of Andrews [An] who proved the case $k=0$ of the following more general

Theorem 2. Assume $P \subset R^{d}$ is a lattice polytope with nonempty interior. Then

$$
f_{k}(P) \ll(\operatorname{Vol} P)^{\frac{d-1}{d+1}},
$$

where the implied constant depends only on $d$.

The result was rediscovered by Arnol'd [Ar] (case $d=2$ ), Konyagin and Sevastyanov [KS], case $d \geq 2, k=0$ with indication to any $k$. W. Schmidt [Sch] proved (2.1) in slightly stronger form. A more general argument of Bárány and Vershik [BV] implies the case $d \geq 2, k=0$. Here we give yet another proof, based on convex geometry and the technique of cap coverings. What we get is a slight improvement over (2.1), which is also indicated in [KS]. A tower (or flag) of the polytope $P$ is a chain of incident faces $F_{0} \subset F_{1} \subset \cdots \subset F_{d-1}$ with $\operatorname{dim} F_{i}=i$. Write $T(P)$ for the number of towers of $P$.

Theorem 3. Under the previous assumptions

$$
T(P) \ll(\operatorname{Vol} P)^{\frac{d-1}{d+1}} .
$$

As clearly $f_{k}(P) \leq T(P),(2.2)$ indeed generalizes (2.1). The proof, however, starts with the case $k=d-1$ of (2.1) and uses, twice, a trick of Andrews later.

\section{Lower bounds and approximation}

W. Schmidt [Sch] asked whether the exponent $\frac{d-1}{d+1}$ in (2.1) is best possible (when $d>2$ ). In the case $d=2$ this is clear from [Ar] and [Sch], Arnol'd also indicates the general case. The lower bounds of Theorem 1 show that the exponent in (2.1), and also in (2.2), is best possible. An argument of the first named author (given 
in [BD]) proves that the average of $f_{0}\left(P_{r}\right)$, over $r \in[R, R+H]$ is of order $R^{d \frac{d-1}{d+1}}$. This is a weaker, or average, version of the case $k=0$ of Theorem 1.

The proof of the lower bounds in Theorem 1 is based on a result from the theory of approximation of (smooth) convex bodies by polytopes. To state what we need, write $\mathscr{C}(D)$ for the collection of convex bodies with $\mathscr{C}^{2}$ boundary and radius of curvature at every point and every direction between $1 / D$ and $D$. (Here $D \geq 1$.) Let $K \in \mathscr{C}(D)$ and assume $P \subset K$ is a convex polytope. Approximation of $K$ by $P$ is measured as the "relative" missed volume, i.e.,

$$
\operatorname{appr}(K, P)=\frac{\operatorname{Vol}(K \backslash P)}{\operatorname{Vol} K} .
$$

The result we need (cf. [G1]) says that for any $K \in \mathscr{C}(D)$ and for any polytope $P \subset K$ having $n$ vertices

$$
\operatorname{appr}(K, P) \gg n^{-\frac{2}{d-1}}
$$

On the other hand, there is a polytope $P \subset K$ with $n$ vertices satisfying

$$
\operatorname{appr}(K, P) \ll n^{-\frac{2}{d-1}} .
$$

Here $\gg$ and $\ll$ depend on $D$ as well. More precise asymptotic information is available on best approximation (cf. [G2]): the constant is const $(d)$ times the $\frac{d+1}{d-1}$ power of the affine surface area of $K$. But we won't need this precision.

The proof of the lower bounds is based on

Theorem 4. For every $d \geq 2$

$$
\operatorname{Vol}\left(r B^{d} \backslash P_{r}\right) \ll r^{d \frac{d-1}{d+1}} .
$$

This implies the case $k=0$ of Theorem 1: Assume $f_{0}\left(P_{r}\right)=n$. By (3.1) and Theorem 4

$$
n^{-\frac{2}{d-1}} \ll \frac{\operatorname{Vol}\left(r B^{d} \backslash P_{r}\right)}{\operatorname{Vol} r B^{d}} \ll r^{d \frac{d-1}{d+1}-d}=r^{-\frac{2 d}{d+1}}
$$

showing that $f_{0}\left(P_{r}\right)=n \gg r^{d \frac{d-1}{d+1}}$ indeed. On the other hand, $f_{0}\left(P_{r}\right) \ll r^{d \frac{d-1}{d+1}}$ from Theorem 1 which together with (3.1) imply that

$$
r^{-\frac{2 d}{d+1}} \ll f_{0}\left(P_{r}\right)^{-\frac{2}{d-1}} \ll \operatorname{appr}\left(r B^{d}, P_{r}\right),
$$

i.e., $P_{r}$ is a "best" aproximating polytope to $r B^{d}$ in the sense of (3.2). So we have

\section{Corollary .}

$$
f_{0}\left(P_{r}\right)^{-\frac{2}{d-1}} \ll \operatorname{appr}\left(r B^{d}, P_{r}\right) \ll f_{0}\left(P_{r}\right)^{-\frac{2}{d-1}} .
$$


A long time ago, C. A. Rogers [R] proved the following analogue of (3.1). For every polytope $P \subset B^{d}$ with $n$ facets

$$
\operatorname{appr}\left(B^{d}, P\right) \gg n^{-\frac{2}{d-1}} .
$$

From this the case $k=d-1$ of Theorem 1 (the lower bound) follows the same way as above. Cases $k=1, \ldots, d-2$ of Theorem 1 need special, and more involved treatment. The proof would be simpler if, for every convex polytope $P$, one would have

$$
f_{k}(P) \geq \min \left\{f_{0}(P), f_{d-1}(P)\right\} .
$$

This would follow from the unimodality conjecture (see [Z]), which is known to be false. But (3.4) may still be true. It is known to hold for simple (and then simplicial) polytopes, see Björner [Bj].

\section{Replacing $B^{d}$ by $K$}

In this section we assume

$$
K \in \mathscr{C}(D) \text { and } 0 \in \operatorname{int} K .
$$

Let $P_{\lambda}$ be the integer convex hull of $\lambda K$, i.e.,

$$
P_{\lambda}=P_{\lambda}(K)=\operatorname{conv}\left(Z^{d} \cap \lambda K\right) .
$$

Here $\lambda$ is large (we keep the letter $r$ for radius of curvature). The questions, and the answers, of the previous sections extend to this case, with the constants implied in $\ll$ depending on $d$ and $D$ :

Theorem 5. Assume $K$ satisfies (4.1). Then, as $\lambda \rightarrow \infty$,

$$
\lambda^{d \frac{d-1}{d+1}} \ll f_{k}\left(P_{\lambda}(K)\right) \ll \lambda^{d \frac{d-1}{d+1}} .
$$

We will indicate, after the proofs for $B^{d}$, how the extension goes.

The generalization of Rogers' result (3.3) to this case has to be stated and proved separately:

Theorem 6. Assume $K$ satisfies (4.1) and $P \subset K$ is a polytope with $n$ facets. Then

$$
\operatorname{appr}(K, P) \gg n^{-\frac{2}{d-1}}
$$

with the implied constant depending only on $d, D$.

Again, the proof of the lower bound in Theorem 1 for $k=1, \ldots, d-2$ would be simpler if the following unusual approximation statement were true.

Conjecture. Assume $K$ satisfies (4.1), $k \in\{1, \ldots d-2\}$ and $P \subset K$ is a polytope with $f_{k}(P)=n$. Then

$$
\operatorname{appr}(K, P) \gg n^{-\frac{2}{d-1}} \text {. }
$$




\section{Proof of Theorem 4}

We start by introducing notation and terminology. Let $p \in Z^{d}$ be a primitive vector, outward normal to the facet $F(p)$ of $P_{r}$. The hyperplane $H(p)=\operatorname{aff} F(p)$ cuts off a small cap $C(p)$ from $r B^{d}$ and

$$
Z^{d} \cap \operatorname{int} C(p)=\emptyset
$$

Let $\rho=\rho(p)$ be the radius of the $(d-1)$-ball $H(p) \cap r B^{d}$ and let $h=h(p)$ be the width, in direction $p$, of the cap $C$. Then

$$
\rho^{2}=(2 r-h) h \text { and so } r h \ll \rho^{2} \ll r h \text {. }
$$

Write $|x|$ for the Euclidean length of $x \in R^{d}$. Letting Area to denote $(d-1)-$ dimensional volume, we have

$$
\text { Area } F(p)=\ell(p)|p| \ll \rho^{d-1}
$$

where $\ell(p)>0 .|p|$ is, in fact, the determinant of the lattice $Z^{d} \cap H(p)$. So

$$
\ell(p) \in \frac{1}{(d-1) !} Z^{d} .
$$

Lemma 1. The contribution to $\operatorname{Vol}\left(r B^{d} \backslash P_{r}\right)$ of the caps $C(p)$ with $h(p) \leq r^{-\frac{d-1}{d+1}}$ is $\ll r^{d \frac{d-1}{d+1}}$.

Proof. Everything that is contained in such a $C(p)$ is also contained in

$$
r B^{d} \backslash\left(r-r^{-\frac{d-1}{d+1}}\right) B^{d}
$$

whose volume is just $\left(r^{d}-\left(r-r^{-\frac{d-1}{d+1}}\right)^{d}\right) \operatorname{Vol} B^{d} \ll r^{d \frac{d-1}{d+1}}$.

From now on we can only consider facets $F(p)$ with

$$
h(p) \geq r^{-\frac{d-1}{d+1}} .
$$

We are going to use the Flatness Theorem (cf. [K], [KL]) saying that the lattice width of a lattice point free convex body (in $R^{d}$ ) is at most $c_{0} d^{2}$ where $c_{0}$ is a universal constant. Applying this to $C(p)$, or rather to int $C(p)$ which is lattice point free by (5.1), we get a primitive vector $q \in Z^{d}$ such that

$$
\max \{q(x-y) x, y \in C(p)\} \leq c_{0} d^{2} .
$$

Case 1: when $h(p) \leq c_{0} d^{2}|p|^{-1}$. In this case $p$ is a flatness direction for $C(p)$ (since consecutive lattice hyperplanes with normal $p$ are at distance $|p|^{-1}$ apart). Then $\rho^{2} \ll r h \ll r|p|^{-1}$ and

$$
\text { Area } F(p)=\ell(p)|p| \ll \rho^{d-1} \ll\left(r|p|^{-1}\right)^{\frac{d-1}{2}},
$$


implying

$$
\ell(p) \ll r^{\frac{d-1}{2}}|p|^{-\frac{d+1}{2}} .
$$

As $\ell(p) \geq \frac{1}{(d-1) !}$ we get $|p| \ll r^{\frac{d-1}{d+1}}$. We write $b=b(d)$ for the implied constant. The lost volume in Case 1 is

$$
\begin{aligned}
& \ll \sum_{p} \operatorname{Area} F(p) h(p) \ll \sum_{p} \ell(p) \ll \sum_{|p| \leq b r \frac{d-1}{d+1}} r^{\frac{d-1}{2}}|p|^{-\frac{d+1}{2}} \\
& \ll r^{\frac{d-1}{2}} \int_{0}^{b r^{\frac{d-1}{d+1}}} x^{-\frac{d+1}{2}} x^{d-1} d x \ll r^{d \frac{d-1}{d+1}}
\end{aligned}
$$

as a simple computation reveals.

Case 2: when $h(p)>c_{0} d^{2}|p|^{-1}$. Then some $q \in Z^{d}$, distinct from $p$, is the flatness direction of $C(p)$.

Assume $C(p)$ is between hyperplanes $q x=\ell_{1}$ and $q x=\ell_{2}$ with $0<\ell_{1}<$ $\ell_{2} \leq|q| r$ and $\ell_{2}-\ell_{1} \leq c_{0} d^{2}$. Set $k_{i}=|q| r-\ell_{i}$ and $x_{i}=k_{i} /|q|,(i=1,2)$. Consider the two-dimensional plane containing $0, q$, and the centre of $C(p)$. We show first, assuming $x_{2}>0$, that $\phi$ (see the figure) gets small as $r$ gets large. Indeed, using (5.4)

$$
\sin \phi=\frac{x_{1}-x_{2}}{2 \rho}=\frac{x_{1}-x_{2}}{2 \sqrt{(2 r-h) h}} \leq \frac{k_{1}-k_{2}}{2|q| \sqrt{r h}} \leq \frac{c_{0} d^{2}}{2|q| \sqrt{r \cdot r^{-\frac{d-1}{d+1}}}} \ll r^{-\frac{1}{d+1}}
$$

since $|q| \geq 1$.

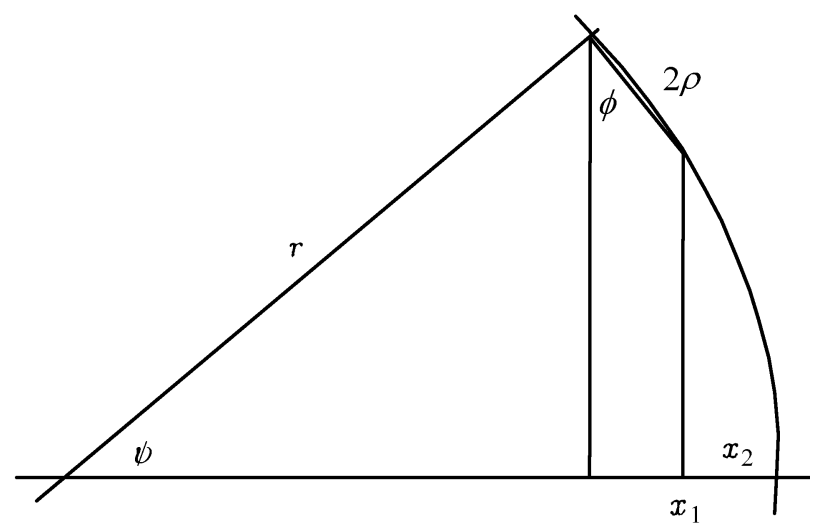

Fig. 1.

As $\phi$ and $\psi$ (see the figure) are almost equal, (5.6) implies

$$
x_{1}=r(1-\cos \psi) \leq r \sin ^{2} \phi \ll r^{\frac{d-1}{d+1}} .
$$

We can estimate $\rho$ from the figure, again. As $\cos \phi>1 / 2$ for large enough $r$, we get 


$$
\begin{aligned}
\rho & <\sqrt{\left(2 r-x_{1}\right) x_{1}}-\sqrt{\left(2 r-x_{2}\right) x_{2}}=\frac{\left(2 r-x_{1}\right) x_{1}-\left(2 r-x_{2}\right) x_{2}}{\sqrt{\left(2 r-x_{1}\right) x_{1}}+\sqrt{\left(2 r-x_{2}\right) x_{2}}} \\
& \leq \frac{\left(2 r-x_{1}-x_{2}\right)\left(x_{1}-x_{2}\right)}{\sqrt{r}\left(\sqrt{x_{1}}+\sqrt{x_{2}}\right)} \leq 2 \sqrt{r} \frac{k_{1}-k_{2}}{|q|} \frac{\sqrt{|q|}}{\sqrt{k_{1}}+\sqrt{k_{2}}} \ll \sqrt{\frac{r}{|q| k_{1}}} .
\end{aligned}
$$

The same estimate follows directly when $x_{2}=0$. From this $h \ll \rho^{2} r^{-1} \ll$ $\left(|q| k_{1}\right)^{-1}$. Now (5.4) shows $k_{1}|q| \ll r^{\frac{d-1}{d+1}}$. Set now $k=\left\lceil k_{1}\right\rceil$. As $p$ is not a flatness direction, $1 \leq k_{1}-k_{2} \leq k_{1}$. So $k \geq 1$ and

$$
k|q| \ll r^{\frac{d-1}{d+1}} .
$$

Collect the $F(p)$ with fixed flatness direction $q$ and fixed $k$ into groups. The missed volume in the corresponding caps is

$$
\ll \sum \operatorname{Area} F(p) h(p) \leq S \max h(p)
$$

where $S$ is the surface area of $r B^{d}$ between hyperplanes $q x=\ell_{1}$ and $q x=\ell_{2}$. Since $\phi$ is small,

$$
\begin{aligned}
S & \leq 2\left(\left[\left(2 r-x_{1}\right) x_{1}\right]^{\frac{d-1}{2}}-\left[\left(2 r-x_{2}\right) x_{2}\right]^{\frac{d-1}{2}}\right) \operatorname{Area} B^{d-1} \\
& \ll\left(\sqrt{\left(2 r-x_{1}\right) x_{1}}-\sqrt{\left.\left(2 r-x_{2}\right) x_{2}\right)}\left[\left(2 r-x_{1}\right) x_{1}\right]^{\frac{d-2}{2}} \ll \sqrt{\frac{r}{|q| k}}\left(\frac{r k}{|q|}\right)^{\frac{d-2}{2}} .\right.
\end{aligned}
$$

where we used the second half of (5.8). Evidently $\max h(p) \leq \rho^{2} / r \ll(|q| k)^{-1}$. We continue (5.9):

$$
\ll \frac{1}{|q| k} \sqrt{\frac{r}{|q| k}}\left(\frac{r k}{|q|}\right)^{\frac{d-2}{2}}=r^{\frac{d-1}{2}}|q|^{-\frac{d+1}{2}} k^{\frac{d-5}{2}} .
$$

This is to be summed for all $k=1,2, \ldots$ and $q \in Z^{d}$ primitive with $k|q| \leq R$ where $R \ll r^{\frac{d-1}{d+1}}$. Then the total missed volume is

$$
\begin{aligned}
& \ll r^{\frac{d-1}{2}} \sum_{k=1}^{R} \sum_{q \in Z^{d}}^{\frac{R}{k}}|q|^{-\frac{d+1}{2}} k^{\frac{d-5}{2}} \ll r^{\frac{d-1}{2}} \sum_{k=1}^{R} \int_{x \in R^{d},|x| \leq \frac{R}{k}}|x|^{-\frac{d+1}{2}} k^{\frac{d-5}{2}} d x \\
& \ll r^{\frac{d-1}{2}} \sum_{k=1}^{R} k^{\frac{d-5}{2}} \int_{0}^{\frac{R}{k}} t^{d-1} t^{-\frac{d+1}{2}} d t \ll r^{\frac{d-1}{2}} \sum_{k=1}^{R} k^{\frac{d-5}{2}}\left(\frac{R}{k}\right)^{\frac{d-1}{2}} \\
(5.10) & =r^{\frac{d-1}{2}} R^{\frac{d-1}{2}} \sum_{k=1}^{R} k^{-2} \ll(r R)^{\frac{d-1}{2}} \ll r^{d \frac{d-1}{d+1}},
\end{aligned}
$$

as one can check easily.

Remark 1. This proof shows the inequality $f_{0}\left(P_{r}\right) \ll r^{d \frac{d-1}{d+1}}$ (from Theorem 1) directly. Actually, it shows the stronger result that

$$
\left|\partial P_{r} \cap Z^{d}\right| \ll r^{d \frac{d-1}{d+1}} .
$$


To see this one has to use the simple fact

$$
\left|F(p) \cap Z^{d}\right| \ll \frac{\operatorname{Area} F(p)}{|p|}
$$

valid for every facet $F(p)$ of $P_{r}$. This gives, in Case 1,

$$
\sum_{p}\left|F(p) \cap Z^{d}\right| \ll \sum_{p} \frac{\operatorname{Area} F(p)}{|p|} \ll \sum_{p} \frac{\rho(p)^{d-1}}{|p|} \ll \sum_{p} r^{\frac{d-1}{2}}|p|^{-\frac{d+1}{2}}
$$

which is $\ll r^{d \frac{d-1}{d+1}}$, according to (5.6). Case 2 is even simpler. Then

$$
\left|F(p) \cap Z^{d}\right| \ll \frac{\operatorname{Area} F(p)}{|p|} \ll \operatorname{Area} F(p) h(p) \ll \operatorname{Vol} C(p)
$$

and (5.9), (5.10) can be applied.

Remark 2. An essentially identical proof works when $B^{d}$ is replaced by $K$ satisfying (4.1). The main difference is that $H(p) \cap \lambda K$ is not a ball. But it is very close to an ellipsoid (since $h(p)$ is very small, less than $\lambda^{-\frac{d-1}{d+1}}$ : this is shown by Lemma 1). This ellipsoid is sandwiched between two concentric balls of radii $\sqrt{\frac{\lambda h}{D}}$ and $\sqrt{2 \lambda h D}$. This shows that the corresponding $\rho$ and Area $F(p)$ can be bounded as in (5.2) and (5.3) with the implied constants depending on $D$ as well.

We elaborate on how to deal with $\phi$ and $\psi$ on the figure. Let $y \in \partial K$ be the point where the outer normal to $K$ is $q$. Then the figure shows the intersection of $P_{\lambda}$ with the two-plane $H$ parallel with $q$, containing the centre of $C(p)$ and the point $\lambda y$. Write $r$ for the radius of curvature at $\lambda y$ of $H \cap \lambda K$. Clearly, $r / \lambda$ is between $1 / D$ and $D$. The boundary of $H \cap \lambda K$, in a neighbourhood of $\lambda y$ is very close to the circle of radius $r$ with centre $\lambda y-r q /|q|$. Now $\phi$ and $\psi$ are the same as on the figure and the estimation of $\sin \phi$ and $x_{1}$ works the same way. ( $h$ on the figure may be different from the depth of the cap $C(p)$ but their ratio is bounded as a function of $D$.)

\section{Auxiliary results}

Let $K$ be a convex body in $R^{d}$. For $x \in K$ and $\lambda>0$ define

$$
M_{K}(x, \lambda)=x+\lambda\{(K-x) \cap(x-K)\} .
$$

This is the $M$-region introduced by Macbeath [M] in 1953. We define two functions $u, v K \rightarrow R$ by

$$
\begin{aligned}
& \text { (6.1) } u(x)=u_{K}(x)=\operatorname{Vol} M_{K}(x, 1) \\
& \text { (6.2) } \quad v(x)=v_{K}(x)=\min \{\operatorname{Vol}(K \cap H) x \in H, H \text { is a halfspace }\} .
\end{aligned}
$$


The set $K(v \geq t)=\{x \in K v(x) \geq t\}$ is evidently convex. So is $K(u \geq t)$ (see $[\mathrm{M}])$ but we will not need this. It follows from the existence of the Löwner-John ellipsoid that $K(v \geq t)$ is nonempty when $t<\frac{1}{2 d !} \operatorname{Vol} K$.

Several properties of these functions, their level sets, and of the $M$-regions are established in $[\mathrm{M}],[\mathrm{ELR}],[\mathrm{BL}],[\mathrm{B}]$. We list those that will be needed later.

Lemma A. ([ELR]) If $M(x, 1 / 2) \cap M(y, 1 / 2) \neq \emptyset$, then $M(x, 1) \subset M(y, 5)$.

Lemma B. (simple) $u(x) \leq 2 v(x)$.

Lemma C. ([BL]) If $v(x) \leq(2 d)^{-2 d} \operatorname{Vol} K$, then $v(x) \leq(3 d)^{d} u(x)$.

Lemma D. ([B]) $K(v \geq t)$ contains no line segment on its boundary (provided $t>0)$.

Lemma E. ([ELR],[B]) Let $C$ be a cap, i.e., $C=K \cap H$ with some halfspace $H$. If $\varepsilon<(2 d)^{-2 d}$ and $C \cap K(v \geq \varepsilon \operatorname{Vol} K)$ is a single point $x$, then $C \subset M(x, 3 d)$ and $\varepsilon \operatorname{Vol} K \leq \operatorname{Vol} C \leq d \varepsilon \operatorname{Vol} K$.

Lemma F. ([BL]) For every convex body $K \subset R^{d}$

$$
\operatorname{Vol} K(v \leq \varepsilon \operatorname{Vol} K) \ll \varepsilon^{\frac{2}{d+1}} \operatorname{Vol} K
$$

with the implied constant depending only on $d$.

When $K \in \mathscr{C}(D)$ and $x$ is close to the boundary of $K, u(x), v(x)$ are easy to estimate. For instance, as we saw it in Remark 2, the boundary of $K$ is very close to an ellipsoid $E$ in the vicinity of $x$, and for ellipsoids $u_{E}(x)$ and $v_{E}(x)$ are simple to determine, and $u_{E}(x)=2 v_{E}(x)$. It follows that, writing $h=h(x)$ for the width of the cap $K \cap H$ giving the minimum in (6.2)

$$
h^{\frac{d+1}{2}} \ll u_{K}(x) \ll v_{K}(x) \ll h^{\frac{d+1}{2}}
$$

with the implied constants depending only on $d, D$.

\section{Proof of Theorems 2 and 3}

Set Vol $P=V$ and define, with clear anticipation, $\varepsilon=\left[3(15 d)^{d} d ! V\right]^{-1}$. Let $F$ be a facet of $P$ (with outer normal $p$ ). Let $x_{F}$ be the point on the boundary of $P(v \geq \varepsilon V)$ where the outer normal coincides with $p$. According to Lemma D, $x_{F}$ is unique. Let $C\left(x_{F}\right)=P \cap\left\{x p\left(x-x_{F}\right) \geq 0\right\}$.

Claim. For distinct facets $F$ and $G$ of $\mathrm{P}$

$$
M\left(x_{F}, 1 / 2\right) \cap M\left(x_{G}, 1 / 2\right)=\emptyset .
$$

Proof. According to Lemma E

$$
\varepsilon V \leq \operatorname{Vol} C\left(x_{F}\right) \leq d \varepsilon V \text { and } C\left(x_{F}\right) \subset M\left(x_{F}, 3 d\right) .
$$


Assume $M\left(x_{F}, 1 / 2\right) \cap M\left(x_{G}, 1 / 2\right) \neq \emptyset$. Lemma A shows then, that $M\left(x_{F}, 1\right) \subset$ $M\left(x_{G}, 5\right)$, and so

$$
F \subset C\left(x_{F}\right) \subset M\left(x_{F}, 3 d\right) \subset M\left(x_{G}, 15 d\right) .
$$

Since $G \subset C\left(x_{G}\right) \subset M\left(x_{G}, 3 d\right) \subset M\left(x_{G}, 15 d\right)$ as well, $M\left(x_{G}, 15 d\right)$ contains $d+1$ affinely independent lattice points: $d$ from $G$ and at least one more form $F$. The volume of their convex hull is at least $1 / d$ !. Thus by Lemma B

$$
\frac{1}{d !} \leq \operatorname{Vol} M\left(x_{G}, 15 d\right) \leq(15 d)^{d} u\left(x_{G}\right) \leq(15 d)^{d} \cdot 2 \varepsilon V=\frac{2}{3 d !},
$$

a contradiction.

So the $M$-regions $M\left(x_{F}, 1 / 2\right)$ are pairwise disjoint. $P(v \leq \varepsilon V)$ contains half of each: the half cut off by the halfspace $p\left(x-x_{F}\right) \geq 0$. Then by Lemma $\mathrm{F}$ (which is a version of the affine isoperimetric inequality)

$$
\sum_{F} \frac{1}{2} \operatorname{Vol} M\left(x_{F}, \frac{1}{2}\right) \leq \operatorname{Vol} P(v \leq \varepsilon V) \ll \varepsilon^{\frac{2}{d+1}} V \ll V^{\frac{d-1}{d+1}} .
$$

On the other hand, by Lemma C

$$
\operatorname{Vol} M\left(x_{F}, 1 / 2\right)=2^{-d} u\left(x_{F}\right) \geq 2^{-d}(3 d)^{-d} v\left(x_{F}\right) \geq(6 d)^{-d} \varepsilon V \gg 1 .
$$

This clearly implies

$$
f_{d-1}(P) \ll V^{\frac{d-1}{d+1}}=(\operatorname{Vol} P)^{\frac{d-1}{d+1}} .
$$

From this we show, using an idea of Andrews, that $f_{0}(P) \ll(\operatorname{Vol} P)^{\frac{d-1}{d+1}}$.

Let $z$ be a vertex of $P$ with neighbouring vertices $w_{1}, \ldots, w_{n}$. Define

$$
P_{z}=\operatorname{conv}\left\{\cup_{1}^{n}\left\{\frac{2}{3} z+\frac{1}{3} w_{i}+\lambda\left(w_{i}-z\right): \lambda \geq 0\right\}\right\}
$$

As $z \notin P_{z}$, there is a facet $F_{z}$ of $P_{z}$ separating them. This facet is of the form $\operatorname{conv}\left\{\frac{2}{3} z+\frac{1}{3} w_{i}\right.$ : some $\left.i\right\}$. Set $Q=\cap P_{z}$ for all vertices $z$ of $P$. Then $F_{z}$ is a facet of $Q$ as well and $F_{z} \neq F_{y}$ for distinct $z, y . Q$ is a lattice polytope in $\frac{1}{3} Z^{d}$ so

$$
f_{0}(P) \leq f_{d-1}(Q) \ll(\operatorname{Vol} Q)^{\frac{d-1}{d+1}} \ll(\operatorname{Vol} P)^{\frac{d-1}{d+1}} .
$$

We are now in a position to prove Theorem 3.

Proof of Theorem 3. We are going to define a polytope $Q \subset P$ which is a lattice polytope in $\frac{1}{s(d)} Z^{d}$ (where $s(d)$ depends only on $d$ ), and a map $f$ from the towers of $P$ to the vertices of $Q$ that maps distinct towers to distinct vertices. This will show

$$
T(P) \leq f_{0}(Q) \ll\left(s^{d} \operatorname{Vol} Q\right)^{\frac{d-1}{d+1}} \ll(\operatorname{Vol} P)^{\frac{d-1}{d+1}} .
$$

The proof is by induction and we start with $d=2$. The vertices of $P$ are $z_{1}, \ldots, z_{n}$ in this order. The vertices of $Q$ will be 


$$
\frac{2}{3} z_{i}+\frac{1}{3} z_{i+1}, \text { and } \frac{1}{3} z_{i}+\frac{2}{3} z_{i+1} \text { for } i=1, \ldots, n \text {. }
$$

The towers of $P$ are $z_{i},\left\{z_{i}, z_{i+1}\right\}$ and $z_{i+1},\left\{z_{i}, z_{i+1}\right\}$. Define

$$
f\left(z_{i},\left\{z_{i}, z_{i+1}\right\}\right)=\frac{2}{3} z_{i}+\frac{1}{3} z_{i+1} \text { and } f\left(z_{i+1},\left\{z_{i}, z_{i+1}\right\}\right)=\frac{1}{3} z_{i}+\frac{2}{3} z_{i+1} .
$$

This is evidently fine; we get $s(2)=3$.

Now for $d \geq 3$. For every facet $F$ of $P$ the inductional hypothesis guarantees the existence of a lattice polytope $Q^{F} \subset F$ (in the lattice $\frac{1}{s(d-1)} Z^{d} \cap$ aff $F$ ) and a mapping

$$
f^{F}\{\text { towers of } F\} \rightarrow\left\{\text { vertices of } Q^{F}\right\} .
$$

Make sure, by contracting $Q^{F}$ suitably if necessary, that $Q^{F} \cap Q^{G}=\emptyset$ for distinct facets $F, G$. It is not hard to see that one can take, as centre of contraction, a point from $\frac{1}{d s(d-1)} Z^{d} \cap \operatorname{conv} F$. Contraction by the factor $1 / 2$ suffices so $Q^{F}$ is a lattice polytope in the lattice $\frac{1}{2 d s(d-1)} Z^{d} \cap$ aff $F$. Set

$$
Q=\operatorname{conv}\left(\cup_{F} Q^{F}\right)
$$

$Q$ is a $\frac{1}{s(d)} Z^{d}$-lattice polytope (with $s(d)=2 d s(d-1)$ ), contained in $P$. To define $f$ let $T_{0} \subset T_{1} \subset \cdots \subset T_{d-1}$ be a tower of $P$. Then $T_{d-1}=F$ for some facet $F$. Define

$$
f\left(T_{0}, \ldots, T_{d-1}\right)=f^{F}\left(T_{0}, \ldots, T_{d-2}\right) \in \operatorname{vert} Q^{F} \subset \text { vert } Q .
$$

\section{Proof of Theorem 6}

In this section the implied constants depend on $d$ and $D$ as well. We assume Vol $K=1$. Then Area $\partial K \gg 1$.

Let $F$ be a facet of $P$ and denote by $x_{F}$ the point where the function $v_{K}$ is maximal on aff $F$. Note that $x_{F}$ need not be contained in $F$. But the cap $C\left(x_{F}\right)$ cut off from $K$ by aff $F$ must have small $\left(\ll n^{-\frac{2}{d+1}}\right)$ volume as otherwise there is nothing to prove. Write $h_{F}$ for the depth of the facet $F$ in $K$; this is the same as the width of the cap $C\left(x_{F}\right)$. As $K \in \mathscr{C}(D)$ and $h_{F}$ is small, (6.3) applies yielding

$$
h_{F}^{\frac{d+1}{2}} \ll u\left(x_{F}\right) \ll v\left(x_{F}\right) \ll h_{F}^{\frac{d+1}{2}} .
$$

Similarly,

$$
h_{F}^{\frac{d-1}{2}} \ll \operatorname{Area}(K \cap \operatorname{aff} F) \ll \operatorname{Area}\left(M\left(x_{F}, 1\right) \cap \operatorname{aff} F\right) \ll h_{F}^{\frac{d-1}{2}} .
$$

Choose a system $y_{1}, \ldots, y_{m} \in\left\{x_{F} F\right.$ a facet $\}$, maximal with respect to the condition that for distinct $i, j$ 


$$
M\left(y_{i}, 1 / 2\right) \cap M\left(y_{j}, 1 / 2\right)=\emptyset .
$$

Half of each $M\left(y_{i}, 1 / 2\right)$ is contained in $K \backslash P$. So with (8.1) we get

$$
\sum_{1}^{m} h_{i}^{\frac{d+1}{2}} \ll \sum_{1}^{m} \frac{1}{2} \operatorname{Vol} M\left(y_{i}, \frac{1}{2}\right) \leq \operatorname{Vol}(K \backslash P) .
$$

On the other hand, by Lemma A, for every facet $F$ of $P$ there is an $i$ such that $M\left(x_{F}, 1\right) \subset M\left(y_{i}, 5\right)$. In this case the outer unit normals to the facets $F$ and $F\left(y_{i}\right)$ cannot differ much. Then $S_{i}$, the total $(d-1)$-volume of the projections of all such facets $F$ onto aff $F\left(y_{i}\right)$ is essentially equal to the $(d-1)$-volume of these facets. So we get, using (8.2) as well,

$$
\text { Area } \partial P=\sum_{F} \text { Area } F \ll \sum_{1}^{m} S_{i} \leq \sum_{1}^{m} \operatorname{Area}\left[\operatorname{aff} F\left(y_{i}\right) \cap M\left(y_{i}, 5\right)\right] \ll \sum_{1}^{m} h_{i}^{\frac{d-1}{2}} .
$$

Of course, Area $\partial P \gg 1$. We combine (8.3), (8.4), and the inequality between the $\frac{d-1}{2}$ and $\frac{d+1}{2}$ means:

$$
\left(\frac{1}{m}\right)^{\frac{2}{d-1}} \ll\left(\frac{\sum h_{i}^{\frac{d-1}{2}}}{m}\right)^{\frac{2}{d-1}} \leq\left(\frac{\sum h_{i}^{\frac{d+1}{2}}}{m}\right)^{\frac{2}{d+1}} \ll\left(\frac{\operatorname{Vol}(K \backslash P)}{m}\right)^{\frac{2}{d+1}}
$$

This gives

$$
\operatorname{appr}(K, P)=\frac{\operatorname{Vol}(K \backslash P)}{\operatorname{Vol} K} \gg m^{1-\frac{d+1}{d-1}}=m^{-\frac{2}{d-1}} \geq n^{-\frac{2}{d-1}},
$$

since $n \geq m$.

Remark 3. The proof works even if the maximal system $y_{1}, \ldots, y_{m}$ is chosen from a subset of the facets, if the total $(d-1)$-volume of these facets is $\gg 1$. This observation will be used in the next section.

\section{Lower bounds for $k=1, \ldots, d-2$}

We show first that most of the surface area of $P_{r}$ comes from facets whose depth $h$ is between $b_{1} r^{-\frac{d-1}{d+1}}$ and $b_{2} r^{-\frac{d-1}{d+1}}$ where $b_{1}<1$ is small, $1<b_{2}$ is large.

Lemma 2. The contribution to the surface area of $P_{r}$ of the facets with $h \leq$ $b_{1} r^{-\frac{d-1}{d+1}}$ is $\ll b_{1}^{\frac{d-1}{2}} r^{d-1}$.

Proof. The surface area of $F(p)$ with $h=h(p) \leq b_{1} r^{-\frac{d-1}{d+1}}$ is at most

$$
\rho^{d-1} \text { Area } B^{d-1} \ll(r h)^{\frac{d-1}{2}} \ll b_{1}^{\frac{d-1}{2}} r^{\frac{d-1}{d+1}} .
$$

The total number of facets is $\ll r^{d \frac{d-1}{d+1}}$, so the surface area in question is indeed

$$
\ll b_{1}^{\frac{d-1}{2}} r^{\frac{d-1}{d+1}} r^{d \frac{d-1}{d+1}}=b_{1}^{\frac{d-1}{2}} r^{d-1} .
$$


Lemma 3. The contribution to the surface area of $P_{r}$ of the facets with $h \geq$ $b_{2} r^{-\frac{d-1}{d+1}}$ is $\ll b_{2}^{-1} r^{d-1}$

Proof. Define $D(p)$ as the set of points $x \in r B^{d}$ such that the segment $[0, x]$ intersects the facet $F(p)$. Clearly, the $D(p)$ are pairwise internally disjoint and their union is $r B^{d} \backslash P_{r}$. Let $y \in F(p)$ be the point closest to $x_{p}$, the centre of the cap $C(p)$. Let $m(p)$ denote the length of the longest segment parallel with $p$ that is contained in $D(p)$. Clearly, this segment starts at $y$.

Claim. $m(p) \gg h(p)$

The claim implies the Lemma as follows. The halfline starting at the origin and containing $y$ intersects the boundary of $r B^{d}$ at $y^{\prime}$. So $\operatorname{conv}\left(F(p) \cup\left\{y^{\prime}\right\}\right) \subset$ $D(p)$ and its volume equals $\frac{1}{d} \operatorname{Area} F(p)$ times the $p$-component of the vector $y^{\prime}-y$. The latter is at least $\frac{1}{2} m(p)$ since $p$ is almost parallel with $y^{\prime}-y$. So, using Theorem 4 ,

$$
\begin{aligned}
r^{d \frac{d-1}{d+1}} & \gg \operatorname{Vol}\left(r B^{d} \backslash P_{r}\right) \geq \sum_{\text {all } p} \operatorname{Vol} D(p) \\
& \geq \sum_{\text {all } p} \frac{1}{2 d} m(p) \operatorname{Area} F(p) \gg \sum_{h(p) \geq b_{2} r^{-\frac{d-1}{d+1}}} h(p) \operatorname{Area} F(p) \\
& \gg b_{2} r^{-\frac{d-1}{d+1}} \sum_{h(p) \geq b_{2} r^{-\frac{d-1}{d+1}}} \operatorname{Area} F(p),
\end{aligned}
$$

which proves the Lemma.

Now for the claim. Set $\rho=\rho(p), m=m(p)$, etc, and $\rho_{1}=\left|y-x_{p}\right|$. If $\rho_{1} \leq \rho \sqrt{1-\frac{1}{d-1}}$, then

$$
m \geq \frac{\rho-\rho_{1}}{\rho} h \geq\left(1-\sqrt{1-\frac{1}{d-1}}\right) h \geq \frac{1}{2(d-1)} h .
$$

and we are done. So suppose $\rho_{1}>\rho \sqrt{1-\frac{1}{d-1}}$.

Write $B_{0}$ for the (d-1)-ball $r B^{d} \cap$ aff $F(p)$. Let $C$ denote the $(d-1)$-cap cut off from $B_{0}$ by the hyperplane orthogonal to $y-x_{p}$ and passing through $y$. The diameter of $C$ is $2 \sqrt{\rho^{2}-\rho_{1}^{2}}<\frac{2}{\sqrt{d-1}} \rho$. $C$ contains $F(p)$ and so it contains $d$ affinely independent vectors $v_{1}, \ldots, v_{d} \in Z^{d}$. The hyperplane aff $F(p)$ is then covered by lattice translates of the parallelotope spanned by $v_{2}-v_{1}, \ldots, v_{d}-v_{1}$ and $x_{p}$ is contained in one of the translates. As it is well-known, this translate has a vertex at distance at most $\frac{1}{2} \sqrt{d-1} \max \left|v_{i}-v_{1}\right| \leq \frac{1}{2} \sqrt{d-1} \operatorname{diam} C<\rho$ from $x_{p}$. So this vertex is in $B_{0}$ and consequently in $F(p)$. Then it cannot be closer to $x_{p}$ than $\rho_{1}$, the shortest distance between $x_{p}$ and $F(p)$ :

$$
\rho_{1} \leq \frac{1}{2} \sqrt{d-1} \max \left|v_{i}-v_{1}\right| \leq \frac{1}{2} \sqrt{d-1} \operatorname{diam} C=\sqrt{d-1} \sqrt{\rho^{2}-\rho_{1}^{2}} .
$$


This shows $\rho_{1} \leq \rho \sqrt{1-\frac{1}{d}}$ and the previous argument applies again:

$$
m \geq \frac{\rho-\rho_{1}}{\rho} h \geq\left(1-\sqrt{1-\frac{1}{d}}\right) h \geq \frac{1}{2 d} h .
$$

Now choose a small $b_{1}=b_{1}(d)$ and a large $b_{2}=b_{2}(d)$ so that half of the surface area of $P_{r}$ comes from facets $F(p)$ satisfying

$$
b_{1} r^{-\frac{d-1}{d+1}} \leq h(p) \leq b_{2} r^{-\frac{d-1}{d+1}} .
$$

Write $\mathscr{F}$ for the collection of these facets. We apply the proof method of Theorem 6, this time with $r B^{d}$ instead of $K$. So choose a system $F_{1}, \ldots, F_{m}$ of facets (from $\mathscr{F}$ ) maximal with respect to the condition that

$$
M\left(y_{i}, 1 / 2\right) \cap M\left(y_{j}, 1 / 2\right)=\emptyset,
$$

where $y_{i}$ is the point where $v$ is maximal on aff $F_{i}$. The previous proof, combined with Remark 3, gives

$$
m \gg r^{d \frac{d-1}{d+1}} \text {. }
$$

Now define

$$
\mathscr{F} j=\left\{F_{i} \in \mathscr{F}: 2^{j} r^{-\frac{d-1}{d+1}} \leq h_{i}<2^{j+1} r^{-\frac{d-1}{d+1}}\right\} .
$$

Clearly $\log b_{1} \leq j \leq \log b_{2}$ implying the existence of a $j$ such that

$$
\mathscr{\mathscr { F } _ { j }} \geq\left(\log \frac{b_{1}}{b_{2}}\right)^{-1} m \gg r^{d \frac{d-1}{d+1}}
$$

Fix such a $j$.

Now let $L$ be a $k$-face of $P_{r}$ and fix a point $x_{L} \in L$. If $L \subset F_{i}$ for some $F_{i} \in \mathscr{T}_{j}$, then the cap $C\left(y_{i}\right)$ lies in a ball with centre $x_{L}$ and radius $2^{\frac{j}{2}+2} r^{\frac{1}{d+1}}$. Indeed, as $x_{L} \in L \subset F_{i} \subset C\left(y_{i}\right)$, the distance between $x_{L}$ and $y_{i}$ is at most $\rho_{i}$. The diameter of $C\left(y_{i}\right)$ is

$$
2 \rho_{i}=2 \sqrt{\left(2 r-h_{i}\right) h_{i}} \leq 2 \sqrt{2 r \cdot 2^{j+1} r^{-\frac{d-1}{d+1}}}=2^{\frac{j}{2}+2} r^{\frac{1}{d+1}} .
$$

Consider now the $M$-regions $M\left(y_{i}, 1 / 2\right)$ for $i$ with $F_{i} \in \mathscr{T}_{j}$. Since they are pairwise disjoint, so are their intersections with the sphere $S_{R}$ of radius $R=r-\frac{9}{8} 2^{j} r^{-\frac{d-1}{d+1}}$, centred at the origin. A straightforward, if tedious, computation shows that $S_{R} \cap M\left(y_{i}, 1 / 2\right)$ contains a spherical cap of radius $2^{\frac{j}{2}-1} r^{\frac{1}{d+1}}$. These caps are all contained in the intersection of $S_{R}$ with the ball of radius $2^{\frac{j}{2}+2} r^{\frac{1}{d+1}}$ (centred at $x_{L}$ ). An easy computation shows that there are at most $8^{d-1}$ such caps. This implies that at most $8^{d-1}$ facets from $\mathscr{T}_{j}$ contain $L$. So the total number of $k$-faces is at least $8^{-(d-1)}\left|\mathscr{T}_{j}\right| \gg m \gg r^{d \frac{d-1}{d+1}}$.

Remark 4. The extension of this estimate to $K \in \mathscr{C}(D)$ from $B^{d}$ is similar to the one outlined in Remark 2. Details are left to the reader. 


\section{References}

[A] G. E. Andrews, A lower bound for the volumes of strictly convex bodies with many boundary points, Trans. Amer. Math. Soc., 106 (1963), 270-279.

[Ar] V. I. Arnold, Statistics of integral convex polytopes, Funk. Anal. Pril., 14 (1980), no. 1, 1-3. (in Russian)

[BB] A. Balog, I. Bárány, On the convex hull of the integer points in a disc, DIMACS Series, Vol 6 (1991) Discrete and Computational Geometry, 39-44.

[BD] A. Balog, J-M. Deshouillers, On some convex lattice polytopes, (to appear) 1998.

[B] I. Bárány, Intrinsic volumes and $f$-vectors of random polytopes, Math. Ann., 285 (1989), 671-699.

[BL] I. Bárány, D. G. Larman, Convex bodies, economic cap coverings, random polytopes, Mathematika, 35 (1988), 274-291.

[BHL] I. Bárány, R. Howe, L. Lovász, On integer points in polyhedra: a lower bound, Combinatorica, 12 (1992), 135-142.

[BV] I. Bárány, A.M. Vershik, On the number of convex lattice polytopes, GAFA Journal, 12 (1992), 381-293.

[Bj] A. Björner, Partial unimodality for $f$-vectors of simplicial polytopes and spheres, Contemp. Math., 178 (1994), 45-54.

[CHKM] W. Cook, M. Hartman, R. Kannan, C. McDiarmid, On integer points in polyhedra, Combinatorica, 12 (1992), 27-37.

[ELR] G, Ewald, D.G. Larman, C.A. Rogers, The direction of the line segments and of the $r-$ dimensional balls on the boundary of a convex body in Euclidean space, Mathematika, 17 (1970), 1-20.

[G1] P. M. Gruber, Aspects of approximation of convex bodies, in: Handbook of convex geometry, North-Holland (1993), 319-345.

[G2] P. M. Gruber, Asymptotic estimates for best and stepwise approximation of convex bodies, I and II, Forum Math., 5 (1993), 281-297 and 521-538.

[J] V. Jarnik, Über Gitterpunkte und konvex Kurven, Math. Z., 2 (1925), 500-518.

[K] A. Khintchine, A qualitative formulation of Kronecker's theory of approximation, Izv. Akad. Nauk SSSR Ser. Mat., 12 (1948), 113-122. (in Russian)

[KL] R. Kannan, L. Lovász, Covering minima and lattice point free convex bodies, Annals of Math. 128 (1988), 577-602.

[KS] S. B. Konyagin, K. A. Sevastyanov Estimation of the number of vertices of a convex integral polyhedron in terms of its volume, Funk. Anal. Pril., 18 (1984), no. 1, 13-15. (in Russian)

[M] A. M. Macbeath, A theorem on non-homogenuous lattices, Annals of Math., 56 (1952), 269-293.

[R] C. A. Rogers, The volume of a polyhedron inscribed in a sphere, J. London Math. Soc., 28 (1953), 410-416.

[Sch] W. Schmidt, Integral points on surfaces and curves, Monatshefte. Math., 99 (1985), 45-82.

[V] I.M. Vinogradov, On the number of integer points on a sphere, Izv. Akad.Nauk SSSR Ser. Mat., 27 (1963), 957-986. (in Russian)

[W] A. Walfisz, Gitterpunkte in mehrdimensionalischen Kugeln, Panstwowe Wydawnictwo Naukowe, Warszawa, 1957.

[Z] G. Ziegler, Lectures on polytopes, Springer 1995. 